\title{
Enoxaparin-induced atraumatic acute compartment syndrome of the thigh: a case report
}

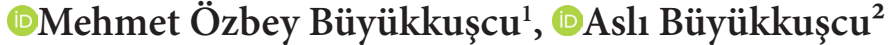 \\ ${ }^{1}$ University of Health Science, Gaziosmanpaşa Training and Research Hospital, Department of Orthopedics and Traumatology, İstanbul, Turkey \\ ${ }^{2}$ University of Health Science, Gaziosmanpaşa Training and Research Hospital, Department of Internal Medicine, İstanbul, Turkey
}

Cite this article as: Büyükkuşcu MÖ, Büyükkuşcu A. Enoxaparin-induced atraumatic acute compartment syndrome of the thigh: a case report. J Health Sci Med 2021; 4(3): 391-393.

\begin{abstract}
In this case, we present a case of atraumatic lateral compartment syndrome of the thigh in a patient undergoing anticoagulant treatment. The patient received high-dose low molecular weight heparin (LMWH) treatment due to the diagnosis of an ischemic cerebrovascular event and developed lateral compartment syndrome of the thigh after the LMWH injection into the thigh; therefore, an emergency fasciotomy was performed. After negative-pressure dressing and monitoring, the wound was closed on the sixth day and the patient was discharged. No complication occurred during the one-month follow-up. Compartment syndrome of the thigh is a rare, but potentially devastating. The purpose of the current report was to highlight this rare, but serious, complication of a routine treatment procedure.
\end{abstract}

Keywords: Compartment syndrome, thigh, atraumatic, anticoagulant treatment, complication

\section{INTRODUCTION}

Anticoagulants are often used both prophylactically and to treat venous thrombosis. Since such treatments increase the susceptibility of the patient to hemorrhage, low-energy trauma may result in disproportionate intracompartmental bleeding and development of compartment syndrome. There is need to perform rapid diagnosis and prompt treatment to prevent this potential disaster (1).

In the literature, compartment syndromes have been reported in association with the use of anticoagulants. The use of these agents has substantially increased due to the reduced need for laboratory monitoring and their convenience for home use (2). Although many studies suggest increased safety and efficacy of LMWH compared to unfractionated heparin, associated bleeding complications are still described (3). Research indicates that minor bleeding is more common than major bleeding complications. In the literature, the incidence of major bleeding in patients treated with enoxaparin has been shown to vary between 4.7 and $5.2 \%(4,5)$. The aim of the current study was to present a rare but potentially destructive compartment syndrome complication that developed under the most frequently preferred anticoagulant therapy.

\section{CASE REPORT}

A 46-year-old female patient presented to the emergency department with a severe headache, nausea, and droopy right eyelid. There was no comorbidity other than migraine and hypertension. The patient was admitted to the neurology clinic with a diagnosis of an ischemic cerebrovascular event, and acetylsalicylic acid (Ecopirin $100 \mathrm{mg} 1 \times 1$ ) treatment was initiated. Dissection of the right vertebral artery was detected on diagnostic angiography. After the cardiology consultation, Ecopirin was stopped and LMWH was started. Enoxaparin (Oksapar, Koçak Farma, Istanbul, TR) was subcutaneously administered at a dose of 0.6 $\mathrm{ml}$ twice a day. On the tenth day, an LMWH injection was administered into the right thigh at the neurology clinic but the patient started to have complaints of severe right thigh pain and swelling that increased after the injection; thus, consultation was requested from clinic of orthopaedic. There was no history of trauma.

The patient's activated partial thromboplastin time, prothrombin time, and international normalized ratio (INR) were found to be normal. Kidney function and liver function tests were also normal. Severe pain in the knee joint passive flexion, tenderness with palpation, and anterolateral hypoesthesia of the right leg were observed. 
Distal pulses (popliteal, tibialis posterior and dorsalis pedis) were palpated. Due to the risk of active bleeding, the cardiovascular surgery department was consulted, and computed tomography (CT) angiography (Figure 1) and magnetic resonance imaging (MRI) (Figure 2) of the lower extremity were undertaken. MRI revealed a $4 \times 5 \times 12 \mathrm{~cm}$ lesion consistent with hematoma in the lateral compartment of the thigh. According to CT angiography evaluated by an experienced musculoskeletal radiologist, there was a millimeter area within the hematoma that constituted the suspicion of active bleeding. However, its relationship with neighboring arterial structures could not be determined. Cardiovascular surgeon stated that vascular intervention was not required.

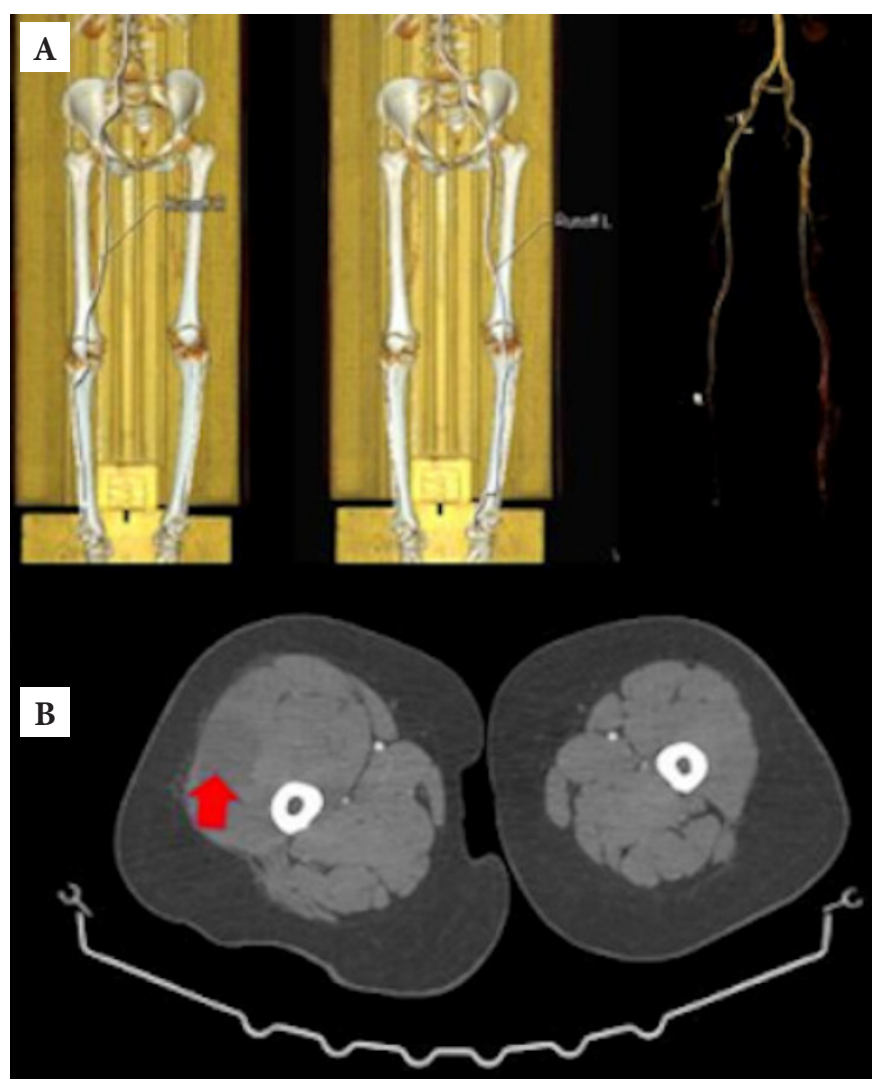

Figure 1. a: Computed tomography angiogram scan views. b: Computed tomography view showing a large hematoma in the right thigh (red arrow).

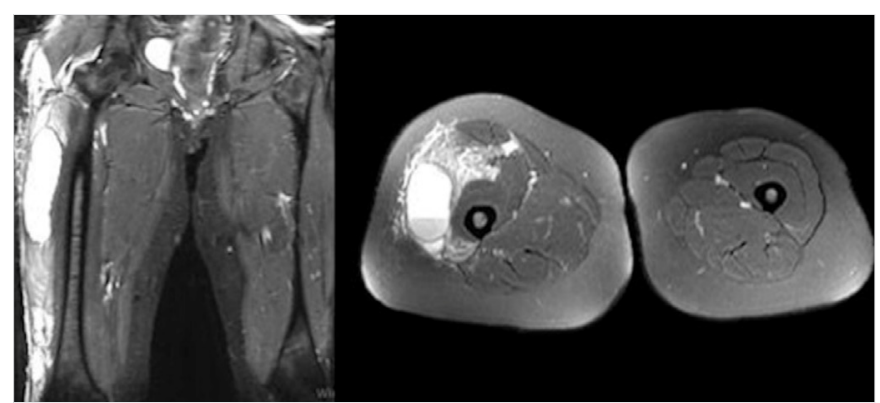

Figure 2. Coronal and axial magnetic resonance image of the thighs, showing a large intramuscular hematoma, as well as widespread muscle edema in the lateral compartment of the left thigh.
After being diagnosed with compartment syndrome, the patient was transferred to the operating room with the indication of an emergency fasciotomy. A lateral compartment fasciotomy was performed through an approximately $10 \mathrm{~cm}$ incision made from the lateral thigh, and the fluid consistent with hematoma was drained. The muscular structure was observed to be completely alive, with no necrotic tissue being detected. The presence of bleeding was checked, and the surgical area was washed with plenty of saline. Suturing was undertaken close to the skin, and the wound was closed with negative-pressure dressing. The patient's hemoglobin level was $10.7 \mathrm{~g} / \mathrm{dl}$ before fasciotomy and $9.8 \mathrm{~g} / \mathrm{dl}$ after the procedure. Postoperatively, the patient's symptoms and signs completely resolved. Upon the development of swelling and pain on the thigh on the second day after fasciotomy, approximation sutures were removed, and approximately $60 \mathrm{cc}$ of hemorrhagic fluid was drained. Anticoagulant treatment with LMWH was stopped after consulting the neurology and cardiovascular surgery departments. The patient was closely followed up, during which her clinical state improved. The wound was closed under local anesthesia on the sixth postoperative day, and the patient was referred to neurology. No event was reported at the first-month follow-up. Patient's consent was obtained for this study.

\section{DISCUSSION}

The importance of this case is that the literature contains only a limited number of cases reported to have developed compartment syndrome of the thigh following an LMWH injection, and it is known that this complication may have devastating consequences. Also, it must be considered compartment syndrome as a differential diagnosis in a patient with a painful limb, especially if they are over-anticoagulated.

Acute compartment syndrome of the thigh is a rare condition. Unlike the forearm or calf, the thigh has much larger compartments that allow for greater increases in volume before splitting pressures become critical. In the literature, it has been reported that compartment syndrome of the thigh most commonly develops after femoral fractures, heavy exercise, blunt trauma, vascular injury, thigh compression, and prolonged positioning (6). In addition, there are three case reports describing non-traumatic compartment syndrome of the thigh in patients receiving LMWH therapy $(1,3,7)$. Reported risk factors for LMWH-induced bleeding include advanced age, use of a high anticoagulant dose, use of other medications such as aspirin, and impaired kidney function (8). 
Limbergetal.(1) reported acase of compartment syndrome of the thigh that developed after an insulin injection into the thigh under enoxaparin treatment. This patient had several of the known risk factors for spontaneous bleeds, including advanced age and impaired renal function. Initially, $300 \mathrm{cc}$ of hemorrhagic fluid was evacuated at the bedside with ultrasound-guided needle aspiration, but after about 1 hour, the swelling and pain recurred, upon which the researchers performed angiography to inspect bleeding and identified an indication for fasciotomy and performed this procedure. Anton et al. (3) reported an elderly patient with renal insufficiency, who spontaneously developed a thigh hematoma while receiving enoxaparin therapy. The patient had a history of hypertension and chronic renal insufficiency, who was under subcutaneous enoxaparin $(70 \mathrm{mg} 2 \times 1)$ treatment with the diagnosis of pulmonary thromboembolism. Anton et al. (3) detected a hematoma in CT performed due to sudden swelling and pain in the left thigh that started on the second day of treatment. It was observed that the hemoglobin level decreased to $7 \mathrm{~g} / \mathrm{dl}$ in a short time. The patient was given urgent fresh frozen plasma and eight units of red blood cells, and discharged with full recovery. Obaid et al. (7) reported that a 5-week old female infant developed persistent sepsis that necessitated surgical removal of a peripherally inserted central catheter adhered to the inferior vena cava with large occlusive clots. Lowmolecular-weight heparin (LMWH), enoxaparin, was started and increased gradually to $2.5 \mathrm{mg}$ every 12 hours. Fifteen days later, a large hematoma developed at the injection site soon after injection. Throughout the next 4 hours the left thigh showed signs of acute compartment syndrome. Enoxaparin was discontinued and protamine was given. After an emergent decompression surgery, the leg healed with residual scarring. The current patient had a risk factor of a high dose of LMWH.

\section{CONCLUSION}

The growing number of cases of spontaneous major bleeding and compartment syndrome associated with the increasing use of LMWH therapy suggests that physicians should be more alert for this complication. LMWH therapy is vital in the treatment of thromboembolic disorders, but patients under LMWH should be monitored with antiXa for long-term treatments. Also, LMWH should not be injected into the thigh, and emergency fasciotomies must be considered in like this cases.

\section{ETHICAL DECLARATIONS}

Informed Consent: Written informed consent was obtained from all participants who participated in this study.
Referee Evaluation Process: Externally peer-reviewed. Conflict of Interest Statement: The authors have no conflicts of interest to declare.

Financial Disclosure: The authors declared that this study has received no financial support.

Author Contributions: All of the authors declare that they have all participated in the design, execution, and analysis of the paper, and that they have approved the final version.

\section{REFERENCES}

1. Limberg RM, Dougherty C, Mallon WK. Enoxaparin-induced bleeding resulting in compartment syndrome of the thigh: a case report. J Emerg Med 2011; 41: 1-4.

2. Titolo P, Milani P, Panero B, Ciclamini D, Colzani G, Artiaco S. Acute compartment syndrome of the arm after minor trauma in a patient with optimal range of oral anticoagulant therapy: a case report. Case Rep Orthop 2014: 980940.

3. Anton E, Marti J. Enoxaparin-associated spontaneous thigh haematoma. Age Ageing 2004; 33: 641-2.

4. Zimmerman DC, Kapoor T, Elfond M, Scott P. Spontaneous compartment syndrome of the upper arm in a patient receiving anticoagulation therapy. J Emerg Med 2013; 44: 53-6.

5. Hogerzeil DP, Muradin I, Zwitser EW, Jansen JA. Acute compartment syndrome of the thigh following hip replacement by anterior approach in a patient using oral anticoagulants. World J Orthop 2017; 18: 964-7.

6. Slade HJT, De Ridder K. A rare cause of acute compartment syndrome in the thigh: a case report. J Surg Case Rep 2020; 28: rjaa546.

7. Obaid L, Byrne PJ, Cheung P. Compartment syndrome in an ELBW infant receiving low-molecular-weight heparins. J Pediatr 2004; 144: 549.

8. Newman PA, Deo S. Non-traumatic compartment syndrome secondary to deep vein thrombosis and anticoagulation. BMJ Case Rep.2014: bcr2013201689. 